\title{
ANÁLISIS DESCRIPTIVO Y DE RELACIÓN ENTRE LA ACTIVIDAD FÍSICA Y EL RENDIMIENTO ACADÉMICO EN JÓVENES ESTUDIANTES DEL CENTRO DE ESPAÑA
}

\author{
Samuel Manzano-Carrasco \\ samuel_manzano1@hotmail.com \\ Sebastián López-Serrano \\ Sara Suárez-Manzano \\ Alberto Ruiz-Ariza \\ Universidad de Jaén
}

Fecha de Recepción: 4 Febrero 2018

Fecha de Admisión: 10 Abril 2018

\section{RESUMEN}

La evidencia sugiere que la práctica de actividad física (AF) se relaciona positivamente con el rendimiento académico en adolescentes. Sin embargo, es escasa la información sobre si las mejoras afectan de la misma manera en todas las asignaturas de acuerdo con el nivel semanal de AF a una intensidad moderada-vigorosa (AFMV). El objetivo fue describir y ver la relación que existe entre la práctica semanal de AFMV con el rendimiento académico de jóvenes estudiantes de entre 12 y 16 años. Se utilizó un diseño descriptivo. AFMV y las calificaciones de los sujetos se evaluaron a través de un cuestionario sociodemográfico. Los resultados mostraron significatividad en los niños donde realizan más AFMV que las niñas durante la semana. Por otro lado, en ambos sexos, fue estadísticamente significativo tener mejores calificaciones en educación física (EF) con el aumento de AFMV semanal. No se encontraron diferencias significativas en las materias de lengua, matemáticas e inglés o francés. Por lo tanto, la práctica de AFMV diaria y semanal es importante para mejorar las calificaciones en la asignatura de EF, aparte de los beneficios que tiene para la salud. Se requieren estudios futuros para contrastar este estudio con una muestra más alta 0 incluso medir algunas variables cognitivas.

Palabras clave: actividad física; rendimiento académico; salud; educación física

\section{ABSTRACT}

Descriptive analysis and relationship between physical activity and academic performance in young students from central Spain.

Evidence suggests that the practice of physical activity $(\mathrm{PA})$ is positively related to academic performance in adolescents. However, there is little information about whether the improvements affect 


\section{ANÁLISIS DESCRIPTIVO Y DE RELACIÓN ENTRE LA ACTIVIDAD FíSICA Y EL RENDIMIENTO ACADÉMICO EN JÓVENES ESTUDIANTES DEL CENTRO DE ESPAÑA}

in the same way in all subjects according to the weekly level of PA at a moderate-vigorous intensity (MVPA). The objective was to describe and see the relationship that exists between the weekly practice of MVPA with the academic performance of young students between 12 and 16 years old. A descriptive design was used. MVPA and the subjects' marks were evaluated through a sociodemographic questionnaire. The results showed significance in the children where they perform more MVPA than the girls during the week. On the other hand, in both sexes, it was statistically significant to have better grades in physical education (PE) with the increase in weekly MVPA. No significant differences were found in the subjects of language, mathematics and english or french. Therefore, the practice of daily and weekly MVPA is a way to improve the marks in the subject of PE, apart from the benefits it has for health. Future studies are required to contrast this study with a higher sample or even measure some cognitive variables.

Keywords: physical activity; academic performance; health; physical education

\section{ANTECEDENTES}

En los últimos años, un creciente número de estudios han demostrado la relación positiva entre la práctica de actividad física (AF) y el rendimiento académico (RA) tanto en niños (Haapala, 2013; Torrijos-Niño et al., 2014) como en adolescentes (Ardoy et al., 2014; Coe et al., 2013). Sin embargo, tan solo un $20 \%$ de los adolescentes cumplen con la recomendación de la Organización Mundial de la Salud (OMS, 2014) de la práctica diaria de actividad física moderada a vigorosa (AFMV) durante al menos 60 minutos. Es por ello, por lo que el ámbito educativo, concretamente, los centros educativos, deben de promocionar la práctica diaria de AF, ya que estudios con adolescentes muestran efectos positivos tanto a nivel fisiológico, (Ruiz-Ariza et al., 2017; Ortega et al., 2008) como a nivel psicológico (Sabiston et al., 2013).

Si nos centramos en el ámbito educativo, el área de educación física (EF) es una de las que debe potenciar y promocionar la práctica de AF diaria tanto dentro como fuera del contexto escolar. Ardoy et al. (2014) han revelado que tanto el número de sesiones como la intensidad de las clases de EF están asociadas con el rendimiento cognitivo del alumnado. De igual manera, Ruiz-Ariza et al. (2017), mostraron que las cualidades físicas que más afectan el rendimiento cognitivo a estas edades son la capacidad cardiorrespiratoria, la coordinación motora y la agilidad-velocidad. Por el contrario, son limitados los estudios que describen los niveles de AFMV de una muestra del centro de España con respecto a su relación con el rendimiento escolar.

Por otro lado, algunos autores como Hillman et al. (2011) y Davis and Cooper (2011), encontraron en sus investigaciones una relación entre la práctica de AF a una intensidad media-moderada con las matemáticas en general y en la aritmética en particular. Sin embargo, hay pocos estudios que se centran en relacionar esta práctica de AFMV con el RA diferenciando por asignaturas o materias, así como dividiendo la muestra por sexos.

Por tanto, se pretende describir y contrastar los datos existentes entre la práctica diaria de AFMV con el RA, que puede ser de gran interés para futuras líneas enfocadas a mejorar diferentes variables de rendimiento cognitivo.

\section{OBJETIVO}

Describir y ver la relación que existe entre la práctica semanal de AFMV con el RA de jóvenes estudiantes. 


\section{METODOLOGÍA}

\section{Participantes}

La muestra de este estudio se realizó por conveniencia, de un instituto de educación secundaria de la provincia de Ciudad Real (Castilla La Mancha, España). Los sujetos participantes eran jóvenes estudiantes de la E.S.0 de una edad comprendida de entre 12 a 16 años.

\section{Instrumentos}

Para la toma de datos se utilizó un cuestionario sociodemográfico, con diferentes ítems donde se recogían diferentes aspectos, concretamente, la cantidad de días a la semana que realizaban AF de al menos 60 minutos, así como las calificaciones del pasado trimestre de cuatro de sus diferentes materias.

\section{Procedimiento}

El presente estudio se llevó a cabo durante la jornada escolar, donde un investigador durante varias mañanas estuvo pasando estos cuestionarios en las diferentes clases del instituto.

\section{Análisis de datos}

Para analizar los datos de nuestra intervención, se usó una correlación bivariada de Pearson y posteriormente se hizo una regresión lineal simple. Las calificaciones de los sujetos se usaron como variables dependientes. El promedio de la AFMV se utilizó como variable independiente. El análisis se realizó diferenciando ambos sexos.

\section{RESULTADOS}

Tras la realización de los correspondientes análisis estadísticos, los resultados muestran que hay significatividad en los niños donde realizan más AFMV que las niñas durante la semana, concretamente un día más con respecto a las niñas $(p<0,001)$. Además, podemos observar que las calificaciones de las niñas son mayores que la de los niños, apareciendo significatividad en la asignatura de Lengua $(p<0,001)$ e Inglés/Francés $\quad(p=0,042)$ (Ver tabla 1).

Tabla 1. Características descriptivas sociodemográficas.

Los datos se presentan como media y desviación típica en variables continuas.

\begin{tabular}{lccc}
\hline \multicolumn{1}{c}{ VARIABLES } & NIÑS & $\begin{array}{c}\text { NIÑOS } \\
(\mathbf{n}=\mathbf{8 7})\end{array}$ & $\begin{array}{c}\boldsymbol{p} \text { - } \\
\text { valor }\end{array}$ \\
& & & \\
\hline Edad (años) & $13,85 \pm 1,239$ & $14,24 \pm 1,406$ & 0,050 \\
MVPA Promedio & $2,31 \pm 1,23$ & $3,37 \pm 1,66$ & $<\mathbf{0 , 0 0 1}$ \\
(días/semana) & & & \\
Matemáticas & 6,79 & 6,57 & 0,418 \\
Lengua & 6,90 & 5,67 & $<\mathbf{0 , 0 0 1}$ \\
Inglés o Francés & 6,06 & 5,48 & $\mathbf{0 , 0 4 2}$ \\
Educación Física & 7,38 & 7,46 & 0,659 \\
\hline
\end{tabular}

En niñas, tras la realización de los análisis estadísticos se obtuvo una correlación positiva y estadísticamente significativas entre el promedio de AFMV y las diferentes calificaciones de la asignatura de lengua $(p=0,027 ; r=0,228)$, de $E F(p=0,004 ; r=0,296)$ y de Matemáticas $(p=0,024 ; r=0,235)$. (Ver tabla 2). 


\section{ANÁLISIS DESCRIPTIVO Y DE RELACIÓN ENTRE LA ACTIVIDAD FíSICA Y EL RENDIMIENTO ACADÉMICO EN JÓVENES ESTUDIANTES DEL CENTRO DE ESPAÑA}

Sin embargo, una vez realizada la regresión, no se muestran valores significativos en las asignaturas de matemáticas, lengua e inglés. En cambio, en la asignatura de EF, es donde se muestra datos significativos, revelando que la niñas que practican más AF a diario y semanalmente, obtienen mejores calificaciones en EF (Ver tabla 3).

Por otro lado, en los niños, el análisis bivariado de Pearson muestra una correlación positiva y estadísticamente significativa ( $p \quad 0,01$ ) entre la calificación de EF y el promedio de AFMV. Sin embargo, en las demás materias no hay valores estadísticos significativos. (Ver tabla 2).

De igual manera que en las niñas, éstos muestran datos significativos solamente en la materia de EF, incidiendo por tanto, que los niños que realizan a diario y semanalmente AF, obtienen mejores calificaciones en esta asignatura. (Ver tabla 3).

Tabla 2. Correlación bivariada de Pearson en función de la AFMV promedio.

\begin{tabular}{cccccc}
\hline & \multicolumn{5}{c}{ CORRELACIÓN } \\
& $\boldsymbol{n}$ & MATEMÁTICAS & LENGUA & INGLES & E.FISICA \\
\hline \multirow{2}{*}{ NIÑAS } & $\mathbf{9 4}$ & $\mathbf{0 . 0 2 4}$ & $\mathbf{0 . 0 2 7}$ & 0.121 & $\mathbf{0 . 0 0 4}$ \\
\hline \multirow{2}{*}{ NIÑ } & $\mathbf{8 7}$ & 0.672 & 0.394 & 0.494 & $<\mathbf{0 . 0 0 1}$ \\
\hline
\end{tabular}

Tabla 3. Regresión lineal en función de la AFMV promedio.

\begin{tabular}{cccccc}
\hline \multicolumn{5}{c}{ REGRESION LINEAL } \\
& $\boldsymbol{n}$ & MATEMÁTICAS & LENGUA & INGLES & E.FISICA \\
\hline \multirow{2}{*}{ NIÑAS } & $\mathbf{9 4}$ & 0.065 & 0.064 & 0.267 & $\mathbf{0 . 0 0 7}$ \\
\hline \multirow{2}{*}{ NIÑOS } & $\mathbf{8 7}$ & 0.758 & 0.416 & 0.852 & $\mathbf{0 . 0 0 1}$ \\
\hline
\end{tabular}

\section{DISCUSIÓN}

Nuestros hallazgos muestran que gracias a la práctica de AFMV diaria y semanal, el rendimiento académico se ve influido de manera positiva, mejorando las calificaciones de la asignatura de EF. Adicionalmente, destacar de nuestros resultados que los niños practican más AFMV que las niñas.

Estudios recientes, revelan que durante la adolescencia, existe aún una alta capacidad para el aprendizaje y el cerebro es aún muy moldeable (Ruiz-Ariza et al., 2017). De esta manera, la práctica diaria de AFMV es un factor puede influir en producir modificaciones estructurales en el cerebro y también, por sus características inherentes a su práctica, puede ayudar a la adquisición de valores y habilidades como una mayor atención o mejor sociabilidad, decisivas para mejorar el rendimiento escolar o su competencia cívica (Petrides et al., 2016; Ruiz-Ariza et al., 2017).

Es por ello, por lo que los centros educativos poseen un papel fundamental para el fomento y promoción de la práctica de AF diaria, ya que varios estudios revelaron que un aumento en la forma física desde la niñez tiene una influencia significativa, con un RA mejor para la adolescencia (Bezold et al., 2014; Kantomaa et al., 2013). Sin embargo, nuestros hallazgos muestran que no afecta de la 
misma manera la práctica de AFMV en todas las asignaturas, ya que éstas pueden verse influidas por muchos factores de confusión, como puede ser el tiempo de estudio y dedicación, el nivel socioeducativo del alumnado, los estudios de la madre, etc. Futuros estudios deben de ir encaminados a contrastar estos datos incluyendo estas variables extrañas.

Por tanto, la práctica de AFMV diaria supone tener efectos positivos en algunas de las calificaciones, como es en el caso de la asignatura de EF conllevando esto a tener un buen rendimiento y éxito académico. Estudios futuros deben de verificar si esta práctica diaria de AFMV conlleva a tener alguna mejora en variables cognitivas relacionadas con el RA, así como para contrastar este estudio con una muestra más alta o tomar las calificaciones de varios cursos académicos.

\section{CONCLUSIONES}

Se concluye que la práctica de AFMV diaria y semanal es un método para mejorar las calificaciones en la asignatura de EF así como para que lo jóvenes se mantengan activos y con un estilo de vida saludable.

\section{REFERENCIAS BIBLIOGRÁFICAS}

Ardoy, D. N., Fernández-Rodríguez, J. M., Jiménez-Pavón, D., Castillo, R., Ruiz, J. R., \& Ortega, F. B. (2014). A physical education trial improves adolescents' cognitive performance and academic achievement: The EDUFIT study. Scandinavian Journal of Medicine \& Science in Sports, 24(1), 52-61.

Bezold, C. P., Konty, K. J., Day, S. E., Berger, M., Harr, L., Larkin, M. \& Stark, J. H. (2014). The effects of changes in physical fitness on academic performance among New York City youth. The Journal of Adolescent Health : Official Publication of the Society for Adolescent Medicine, 55(6), 774-781.

Coe, D. P., Peterson, T., Blair, C., Schutten, M. C., \& Peddie, H. (2013). Physical fitness, academic achievement, and socioeconomic status in school-aged youth. Journal of School Health, 83(7), 500-507.

Davis, C. L., \& Cooper, S. (2011). Fitness, fatness, cognition, behavior, and academic achievement among overweight children: do cross-sectional associations correspond to exercise trial outcomes? Preventive medicine, 52, 65-69.

Hillman, C. H., Kamijo, K. \& Scudder, M. (2011). A review of chronic and acute physical activity participation on neuroelectric measures of brain health and cognition during childhood. Preventive medicine, 52, 21-28.

Kantomaa, M. T., Stamatakis, E., Kankaanpää, A., Kaakinen, M., Rodriguez, A., Taanila, A., \& Tammelin, T. (2013). Physical activity and obesity mediate the association between childhood motor function and adolescents' academic achievement. Proceedings of the National Academy of Sciences of the United States of America, 110(5), 1917-1922

Haapala, E. A. (2013). Cardiorespiratory fitness and motor ski- Ils in relation to cognition and academic performance in children. A review. Journal of Human Kinetics, 36, 55-68.

Organización Mundial de la Salud. (2014). Recomendaciones mundiales sobre actividad física para la salud. Ginebra: La Organización

Ortega, F. B., Ruiz, J. R., Castillo, M. J., \& Sjöström, M. (2008). Physical fitness in childhood and adolescence: a powerful marker of health. International Journal of Obesity, 32(1), 1-11

Petrides, K. V., Mikolajczak, M., Mavroveli, S., Sanchez-Ruiz, M. J., Furnham, A, y Perez-Gonzalez, J. C. (2016). Developments in Trait Emotional Intelligence Research. Emotion Review, 8(4), 335-341. 
Ruiz-Ariza, A., Grao-Cruces, A., de Loureiro, N. E. M., y Martínez-López, E. J. (2017). Influence of physical fitness on cognitive and academic performance in adolescents: A systematic review from 2005-2015. International Review of Sport and Exercise Psychology, 10(1), 108-133.

Torrijos-Niño, C., Martínez-Vizcaíno, V., Pardo-Guijarro, M. J., García-Prieto, J. C., Arias-Palencia, N. M., \& Sánchez-López, M. (2014). Physical fitness, obesity, and academic achievement in schoolchildren. The Journal of Pediatrics, 165(1), 104-109 